\title{
The perks of a Zeiss Libra 120 plus TEM - First steps towards electron diffraction
}

\author{
Christoph Grininger ${ }^{1}$, Gerhard Hofer ${ }^{2}$, Hongyi $\mathrm{Xu}^{2}$, Xiaodong Zou ${ }^{2}$, Tea Pavkov-Keller ${ }^{1}$ \\ ${ }^{1}$ University of Graz, Graz, Austria; \\ ${ }^{2}$ Stockholm University, Stockholm, Sweden; \\ christoph.grininger@uni-graz.at
}

\begin{abstract}
A well-known bottleneck in the structural characterization of macromolecules with X-ray diffraction is crystallization. Often the needed crystal size cannot be achieved despite extensive optimization of crystallization conditions. Nevertheless, the yield of sea urchin like needle clusters, microcrystals and almost two-dimensional platelets is a silver lining. Those crystals - too small for X-ray crystallography - could be applied to microcrystal electron diffraction (MicroED) methods.
\end{abstract}

3D electron diffraction (ED) is an uprising method for the structural characterization of nanocrystalline materials. ED has recently been applied to beam-sensitive materials like macromolecular crystals. Although there are also drawbacks for this method in protein crystallography, we used the spirit of the nanocrystallography revolution and started with first experiments on our transmission electron microscope.

So far we were able to acquire knowledge for the basic workflow for data collection with our instrument setup, a Zeiss Libra 120 plus TEM with an OMEGA energy filter and a TVIPS TemCam-XF416(ES) detector. We collected continuous rotation electron diffraction data for the zeolite ZSM-5 and protein nanocrystals (lysozyme) under cryo conditions.

With these fundamental achievements we are on track to apply MicroED to more challenging crystals and also solve novel protein structures in the future.

Keywords: electron diffraction, TEM, protein ED 